\title{
Weeds detection efficiency through different convolutional neural networks technology
}

\author{
Adil Tannouche', Ahmed Gaga², Mohammed Boutalline ${ }^{3}$, Soufiane Belhouideg ${ }^{2}$ \\ ${ }^{1}$ Laboratory of Engineering and Applied Technology (LITA), Higher School of Technology (EST-BM), \\ Sultan Moulay Slimane University, Béni Mellal, Morocco \\ ${ }^{2}$ Research Laboratory of Physics and Engineers Sciences, Polydisciplinary Faculty (FP-BM), Sultan Moulay Slimane University, \\ Béni Mellal, Morocco \\ ${ }^{3}$ Systems Engineering Laboratory, National Schools of Applied Sciences (ENSA-BM), Sultan Moulay Slimane University, \\ Béni Mellal, Morocco
}

\section{Article Info \\ Article history: \\ Received Mar 4, 2021 \\ Revised Jul 16, 2021 \\ Accepted Aug 1, 2021}

\section{Keywords:}

Convolutional neural network

Deep learning

Precision agriculture

Vision machine

Weed detection

\begin{abstract}
The preservation of the environment has become a priority and a subject that is receiving more and more attention. This is particularly important in the field of precision agriculture, where pesticide and herbicide use has become more controlled. In this study, we propose to evaluate the ability of the deep learning (DL) and convolutional neural network (CNNs) technology to detect weeds in several types of crops using a perspective and proximity images to enable localized and ultra-localized herbicide spraying in the region of Beni Mellal in Morocco. We studied the detection of weeds through six recent CNN known for their speed and precision, namely, VGGNet (16 and 19), GoogLeNet (Inception V3 and V4) and MobileNet (V1 and V2). The first experiment was performed with the CNNs architectures from scratch and the second experiment with their pre-trained versions. The results showed that Inception V4 achieved the highest precision with a rate of $99.41 \%$ and $99.51 \%$ on the mixed image sets and for its version from scratch and its pre-trained version respectively, and that MobileNet V2 was the fastest and lightest with its size of $14 \mathrm{MB}$.
\end{abstract}

This is an open access article under the CC BY-SA license.

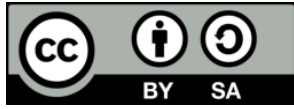

\section{Corresponding Author:}

Adil Tannouche

Laboratory of Engineering and Applied Technology (LITA), Higher School of Technology (EST-BM),

Sultan Moulay Slimane University

Ecole Supérieure de Technologie de Béni Mellal, BP 591, 23000, Béni Mellal, Morocco

Email: tannouche@gmail.com

\section{INTRODUCTION}

Nowadays, artificial intelligence (AI) has made its way into our daily lives with the emergence of embedded systems and internet of things (IoT). Thus, we find it particularly in terms of image processing and recognition. From improving the quality of photos taken by smartphones to security, access control, environmental recognition, and analysis for autonomous vehicles to medical diagnostics, AI is everywhere. Embedded systems are always called upon to analyze more and more large and resource-intensive images.

In precision agriculture and during the last decade, several technologies have been developed to detect weeds and achieve localized and selective spraying. In our previous work, we have combined the Haar-like features with the AdaBoost algorithm to achieve the real time weeds detection in the inter-row of different crops [1]. Also, we have developed a new adjacency descriptor for the selection of weeds (monocot or dicot) to achieve the real time selective spraying [2]. 
In terms of machine learning, several other supervised and unsupervised learning algorithms have been developed for vegetation segmentation. In the supervised learning methods, we cite the use of random forest [3], convolutional neural network (CNN) [4], decision trees [5], support vector machines [6], back propagation neural network [7], and Bayesian classifier [8]. Unsupervised methods focus essentially on Kmeans clustering [9] and K-means clustering based on particle swarm optimization (PSO) [10]. Recently, Wang et al. [11] and Liakos et al. [12] present a detailed review of several artificial vision techniques applied to weed detection.

In agriculture, several problems have been solved by these developments. Recently, the authors have proposed and tested the use of DL in CNNs for tomato plant disease classification [13]. Zaki et al. [14] confirmed that MobileNet V2 correctly classified various tomato plant diseases from leaf images. This finding was confirmed in [15] where the authors concluded that the deep learning (DL) model outperforms the Support vector machine (SVM) by a fairly substantial margin in terms of classification accuracy.

Neural networks and particularly the $\mathrm{CNN}$ are in great demand for image classification, object recognition, face recognition and fine-grained classification. In addition, these networks perform convolutions: Very expensive operations in terms of computation and memory. Image classification, or even object recognition, in embedded systems therefore represents a major challenge due to material constraints.

In this study, we propose to evaluate the ability of the DL and CNN technology to detect weeds in several types of crops to enable localized and ultra-localized herbicide spraying. We present two datasets of RGB images from different cultivated plots. These datasets present regions of interest of $2 \times 3$ and $0.5 \times 0.5 \mathrm{~m}^{2}$ collected in the region of Béni Mellal in Morocco. Then, we studied the detection of weeds through six recent CNN known for their speed and precision, namely, VGGNet (16 and 19), GoogLeNet (Inception V3 and V4) and MobileNet (V1 and V2).

\section{RESEARCH METHOD}

In this section, we describe the details of the implemented CNNs for weed detection in RGB images of different crops. This procedure is divided into four steps: acquisition, learning, classification, and data evaluation. These steps are detailed below.

\subsection{Data acquisition}

In this research, two sets of images of several cereal and vegetable crops were used to develop a reliable and low-cost system able to detect and spray weeds in real time. The first set of images comes from an inclined camera mounted at the front of the spray tractor and allows to capture a tilted region of interest of about $2 \times 3 \mathrm{~m}^{2}$ used to enable localized herbicide spraying. The second set of images comes from a vertical camera allowing capturing a proximity region of interest of about $0.5 \times 0.5 \mathrm{~m}^{2}$ used to enable ultra-localized herbicide spraying. The concept of our real-time spray system is shown in Figure 1.

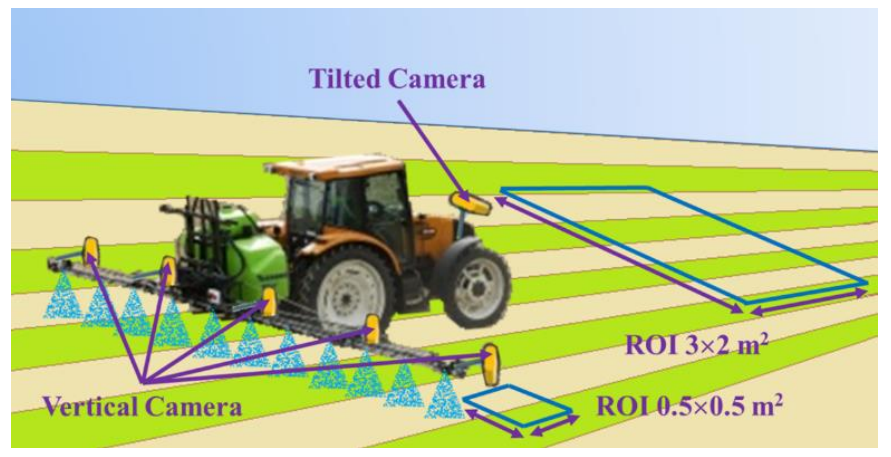

Figure 1. Real-time spraying system concept

Tilted images: These images are derived from video sequences from several cultures for real-time processing [1]. For this current study, each image is first cropped and masked to keep only the region of interest (ROI) of about $3 \times 2 \mathrm{~m}^{2}$ in front of the moving tractor. Then, it is put in an output format as shown in Figure 2. Vertical images: These proximity images are taken vertically to better detect, recognize and classify weeds in the rows and line spacing of different crops [2]. In the present study, these images show ROI of about $0.5 \times 0.5 \mathrm{~m}^{2}$ of several crops. Then, each image is cropped to an output format as shown in Figure 3. 

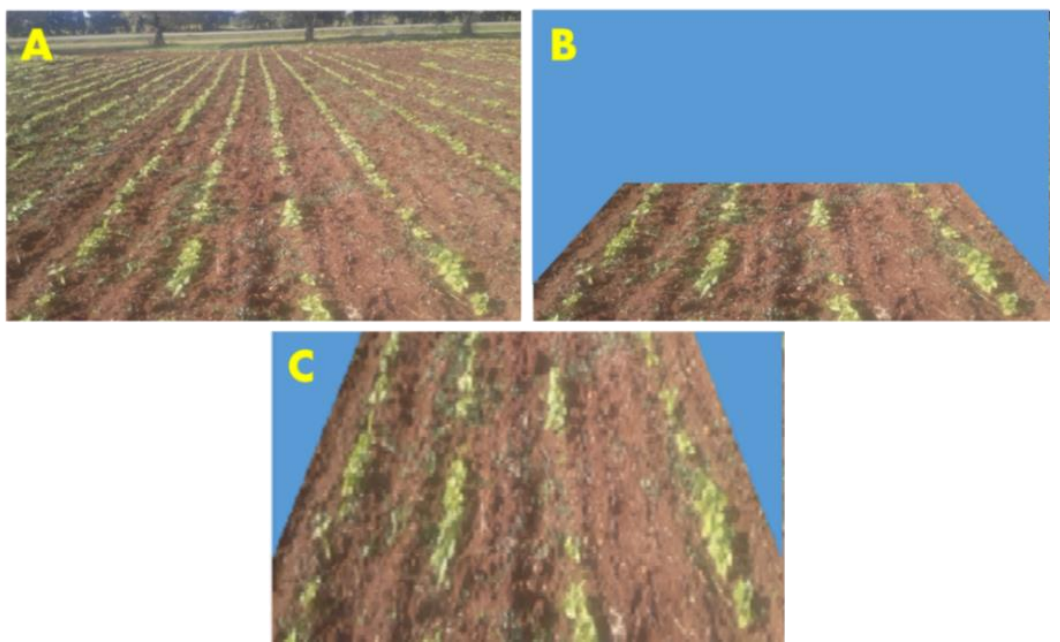

Figure 2. Tilted image of infested bean crop: original image (A), ROI (B) and output format (C)
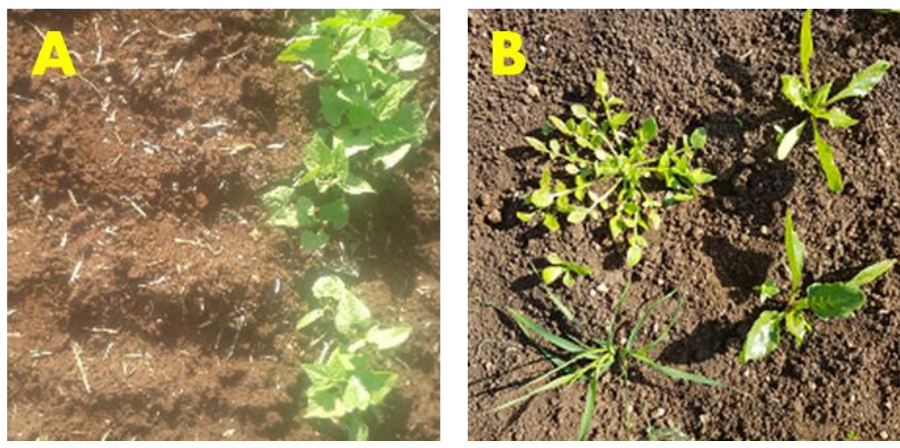

Figure 3. Proximity images: sound bean crop (A) and infested beet crop (B)

These images were taken at different times of the day and on different cultivated plots. We ensured that a wide range of content was covered in the non-weed class images. Also, we included in the weed class several images with visual similarities to those of the non-weed class thus making the task of classification more difficult.

In total, each set of images consisted of 1000 images composed of $50 \%$ images of healthy crops (negative class "0") and 50\% images of crops infested with weeds (positive class "1"). Then we proceeded to increase the number of images by modifying and adjusting the image sharpness, brightness and contrast using the image processing software (IrfanView version 4.54; http://www.Irfanview.com). This process is commonly used for more effective training with small datasets. Consequently, the size of each dataset increased from 1000 images to 10,000 patches. In this way, we constructed three datasets (G1, G2 and G3) composed of tilted, proximity and mixed images, respectively. Each dataset consisted of 8000 patches for training, 1000 patches for validation and 1000 patches for testing.

\subsection{Data training}

Through the sets of images were previously described, the performances of the following CNNs were evaluated for the problem of weeds detection in the different crops.

\subsubsection{CNN architecture}

The CNN architecture is based on a deep neural network structure. It consists of several layers, including convolution, ReLU, pooling, flattening, and fully connected. in the end, a probabilistic distribution is used to generate the classes as shown in Figure 4. In order to determine the content of an image, it goes through two phases: i) the feature extraction phase, consisting of several layers of successive convolutions, which allows decoding certain characteristics of the image; ii) the classification phase predicts the class of the input image (a house, a car, an animal). 
In our case, the number of output classes is reduced to two classes. The first class is the weed class and the second class is the weed-free class. AlexNet [16] was the first emerging CNN model, it demonstrated that the notion of CNN offers a good alternative to solve different image recognition problems. Following this model, many other CNN models have been developed, for example, VGGNet [17], GoogLeNet [18], and MobileNets [19]. Every new version tries to further improve the performance of the CNN in terms of size, accuracy, and speed.

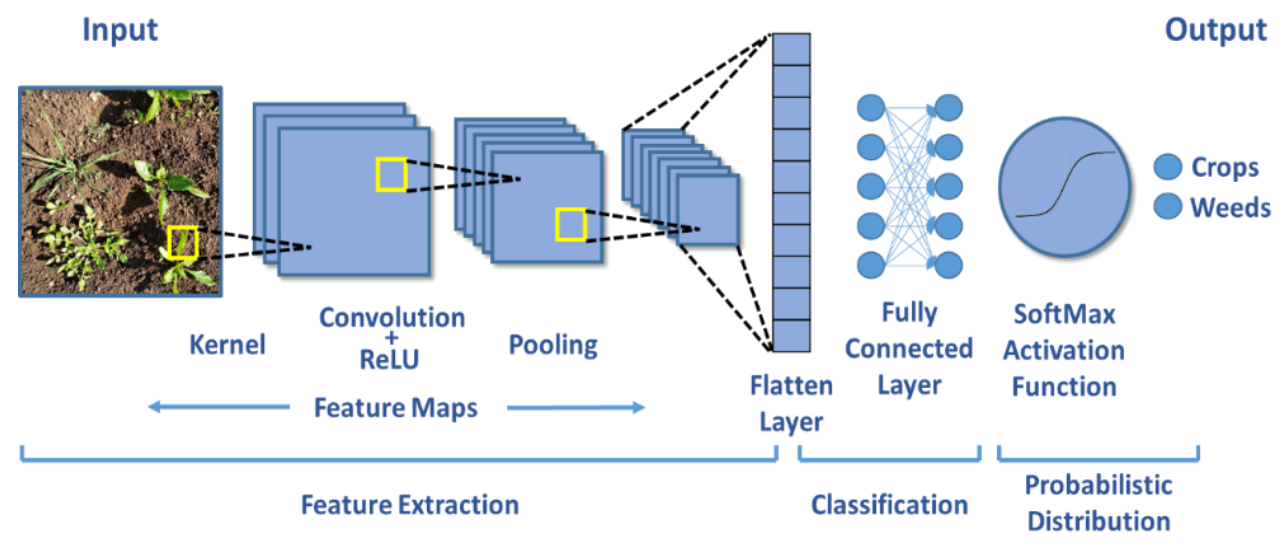

Figure 4. CNN architecture

\subsubsection{VGGNets}

VGGNet is a CNN proposed by Simonyan and Zisserman in 2014 [17]. The VGG network has five versions, of which VGG16 and VGG19 are the most famous. VGG16 has thirteen convolutional layers and three fully connected layers while VGG 19 has sixteen convolutional layers and three fully connected layers. In these two versions, VGG consists of two fully connected layers with 4096 channels in each layer, followed by another fully connected layer with 1000 channels to predict 1000 classes. The last fully connected layer uses the SoftMax layer for classification purposes. Due to its deepest layer, VGG19 performs better than VGG16, but this superiority is gained at the detriment of size.

\subsubsection{GoogLeNet}

GoogLeNet is a CNN created by Szegedy et al. [18]. The first version, called Inception V1, has twenty-two convolution layers augmented by an additional layer known as the Inception module. These builds constructions of different sizes for each convolution node $(1 \times 1,3 \times 3$, and $5 \times 5)$ and $3 \times 3$ max pooling node. Inception V1 won the ILSVRC14 competition ahead of VGGNets.

Following this success, other versions were developed to increase the performances. Thus, the second version, called Inception V2 [20], uses the batch normalization technique to improve the learning performance. The third version, Inception V3 [21], reduced the module of Inception by factoring in the convolution node to improve the speed. The last version is Inception V4 [22]. It adopts a more uniform architecture and uses more inception modules.

\subsubsection{MobileNet}

MobileNet [19] is a reduced CNN destined principally for mobile devices. The basic idea is to replace the classical convolution with two separate processes (depthwise and pointwise separable convolution). Depthwise reduces the length and width dimensions and pointwise reduces the filter in the depth direction [23], [24]. MobileNet has a higher level of performance compared to other CNNs while maintaining a reduced size. MobileNet has a higher level of performance compared to other CNNs while maintaining a reduced size. Given these characteristics, this CNN is ideal for embedded systems. MobileNet V2 is a new version introduced in 2018 [25]. Two features have improved the linear bottleneck and the inverted residual block. MobileNet V2 has become smaller with a reduced number of parameters from 4.2 to 3.4 $\mathrm{M}$ at equivalent performance with its first version.

\subsection{Evaluation de performances}

The evaluation of the weed detection ability in these different studied CNNs is performed using the following metrics: Sensitivity (or recall) (1) reflecting the degree of accuracy of positive examples, 
Specificity (2) corresponding to the conditional probability of true negatives for a subclass, Accuracy (3) which is commonly used to evaluate the accuracy, Precision (4) informing about robustness and F1-Score (5) which at a high value indicates that the performance of the CNN is better in the positive class.

$$
\begin{aligned}
& \text { Sensitivity (Recall) }=\frac{T P}{T P+F N} \\
& \text { Specificity }=\frac{T N}{T N+F P} \\
& \text { Accuracy }=\frac{T P+T N}{T P+T N+F P+F N} \\
& \text { Precision }=\frac{T P}{T P+F P} \\
& F 1-\text { Score }=2 \frac{\text { Precision' Sensitivity }}{\text { Precision +Sensitivity }}
\end{aligned}
$$

Where, TP is number of true positives or positives cases that are positives and classified as positives. TN is number of true negatives or negative cases that are negative and classified as negative. FP is number of false positives, defined by the negative instances that are incorrectly classified as positive cases. FN is number of false negatives, defined by the positive instances that are incorrectly classified as negative cases.

\section{RESULTS AND DISCUSSION}

In this study, an evaluation of a selection of state-of-the-art CNNs, in both scratch and pre-trained versions, for weed detection using a perspective and proximity images of several crop types was performed. The objective of this research was to study these different CNNs by comparing their performance in terms of accuracy, precision, sensitivity, specificity, and the F1-Score measured. All experiments were run on the same machine as shown in Table 1 using Python 3.7, TensorFlow and the Keras library.

All CNNs are trained from scratch without using any pre-trained models, they have been trained for twenty epochs to get a precise idea about their real accuracy, training time, runtime, and size. Note that an epoch represents the set of iterations necessary for the neural network to perform a deep learning pass. Table 2 shows the resolution of the input images, we also find there the resulting size, training time and test time for each CNN trained from scratch on mixed images set. These results remain practically the same for the tilted images set and the proximity images set. We also note that the CNN MobileNet V2 is the lightest and the fastest considering its smallest size, learning and execution time as it got only $14 \mathrm{~GB}, 27$ and $7 \mathrm{~min}$, respectively.

Table 1. Machine specifications

\begin{tabular}{cc}
\hline Components & Specifications \\
\hline Processor & Intel Core i7-7700 CPU @ 3.60 GHz \\
Memory & $16 \mathrm{~Gb}$ \\
Graphics & GeForce GTX 1070 X 8 Gb \\
Operating System & Windows 10, 64 bits \\
\hline
\end{tabular}

Table 2. Features of CNNs formed from scratch

\begin{tabular}{ccccc}
\hline CNN & Input image (Pixel) & Size (MB) & Training Time (Min.) & Test Time (Min.) \\
\hline MobileNet V1 & $224 \times 224$ & 16 & 86 & 8.15 \\
MobileNet V2 & $224 \times 224$ & 14 & 71 & 7.51 \\
Inception V3 & $299 \times 299$ & 84 & 119 & 10.12 \\
Inception V4 & $299 \times 299$ & 163 & 151 & 17.25 \\
VGG 16 & $240 \times 240$ & 528 & 201 & 29.01 \\
VGG 19 & $240 \times 240$ & 548 & 218 & 31.53 \\
\hline
\end{tabular}

Tables 3, 4 and 5 show the performances measured for the different CNNs, formed from scratch, on the sets of inclined, proximities and mixed images, respectively. These different CNNs of this study obtained the best performances on the set of proximity images, then the set of mixed images and finally the set of tilted images. This is because the pictures nearby show more detail. 
As a result, the vegetation is clear and easily differentiated in the agricultural scene, thus weeds are better distinguished in the rows and between rows of crops. At this point, the CNN Inception V4 obtained the highest scores for precision, sensitivity, specificity, accuracy, and F1-Score for all three sets of images. Thus, it displayed respectively $99.31 \%, 99.03 \%, 99.31 \%, 99.18 \%$ and $99.18 \%$ for the tilted images, $99.55 \%$, $99.42 \%, 99.45 \%, 99.45 \%$ and $99.49 \%$ for the proximity images and $99.50 \%, 99.27 \%, 99.54 \%, 99.41 \%$ and $99.41 \%$ for the mixed images.

In accuracy Inception V4 is followed by Inception V3, MobileNet V2, MobileNet V1, VGG 19 and finally VGG 16 which obtained $98.44 \%, 98.37 \%$ and $98.36 \%$ respectively for the sets of tilted, proximity and mixed images. But in general, as can be seen from these statistics, all of these CNNs in this study recorded excellent weed detection results on the three sets of images namely tilted, proximity and mixed images.

Table 3. Measured performance (\%) of CNNs formed from scratch on the tilted images set

\begin{tabular}{ccccccc}
\hline Metric & VGG 16 & VGG 19 & Inception V3 & Inception V4 & MobileNet V1 & MobileNet V2 \\
\hline Precision & 97.74 & 97.81 & 99.22 & 99.31 & 98.79 & 98.99 \\
Sensitivity & 97.38 & 97.51 & 98.99 & 99.03 & 98.49 & 98.53 \\
Specificity & 98.88 & 98.89 & 99.13 & 99.31 & 99.01 & 99.17 \\
Accuracy & 98.14 & 98.21 & 99.07 & 99.18 & 98.76 & 98.86 \\
F-Score & 97.57 & 97.67 & 99.11 & 99.18 & 98.65 & 98.77 \\
\hline
\end{tabular}

Table 4. Measured performance (\%) of CNNs formed from scratch on the proximity images set

\begin{tabular}{ccccccc}
\hline Metric & VGG 16 & VGG 19 & Inception V3 & Inception V4 & MobileNet V1 & MobileNet V2 \\
\hline Precision & 98.14 & 98.21 & 99.52 & 99.55 & 99.19 & 99.23 \\
Sensitivity & 98.01 & 98.11 & 99.39 & 99.42 & 99.23 & 99.21 \\
Specificity & 98.71 & 98.99 & 99.41 & 99.45 & 99.43 & 99.47 \\
Accuracy & 98.37 & 98.56 & 99.41 & 99.45 & 99.34 & 99.35 \\
F-Score & 98.08 & 98.17 & 99.46 & 99.49 & 99.22 & 99.23 \\
\hline
\end{tabular}

Table 5. Measured performance (\%) of CNNs formed from scratch on the mixed images set

\begin{tabular}{ccccccc}
\hline Metric & VGG 16 & VGG 19 & Inception V3 & Inception V4 & MobileNet V1 & MobileNet V2 \\
\hline Precision & 98.01 & 97.96 & 99.32 & 99.50 & 98.83 & 99.12 \\
Sensitivity & 97.65 & 97.60 & 99.09 & 99.27 & 98.53 & 98.66 \\
Specificity & 99.15 & 99.10 & 99.23 & 99.54 & 99.05 & 99.30 \\
Accuracy & 98.36 & 98.51 & 99.17 & 99.41 & 98.80 & 98.99 \\
F-Score & 97.84 & 98.04 & 99.21 & 99.41 & 98.68 & 98.90 \\
\hline
\end{tabular}

Table 6 shows the results of detection of weeds on the set of mixed images obtained by these same CNNs in their pre-trained version. These results are similar to other results obtained with CNNs formed from scratch. Unsurprisingly, the CNN Inception V4 is the best performing. It scored 99.60\%, 99.37\%, 99.64\%, $99.51 \%$ and $99.49 \%$ in terms of Precision, Sensitivity, Specificity, Accuracy, and F1-Score, respectively. In its pre-trained version, it had an accuracy of $99.51 \%$ on the set of mixed images. This shows the effectiveness of using the pre-trained and fine-tuned CNNs version in weeds detection. VGG 16 remains the least efficient among the CNNs studied, with scores of $98.03 \%, 97.67 \%, 99.17 \%, 98.43 \%$ and $97.86 \%$ obtained in terms of precision, sensitivity, specificity, accuracy, and F1-Score, respectively. These results confirm that all of the CNNs in this study recorded excellent weed detection performance on all of the image sets used.

Table 6. Measured performance (\%) of Pre-trained CNN on the mixed images set

\begin{tabular}{ccccccc}
\hline Metric & VGG 16 & VGG 19 & Inception V3 & Inception V4 & MobileNet V1 & MobileNet V2 \\
\hline Precision & 98.03 & 98.14 & 99.32 & 99.60 & 98.95 & 99.20 \\
Sensitivity & 97.67 & 97.78 & 99.09 & 99.37 & 98.65 & 98.74 \\
Specificity & 99.17 & 99.28 & 99.23 & 99.64 & 99.17 & 99.38 \\
Accuracy & 98.43 & 98.54 & 99.17 & 99.51 & 98.92 & 99.07 \\
F-Score & 97.86 & 97.97 & 99.22 & 99.49 & 98.81 & 98.98 \\
\hline
\end{tabular}

MobileNet V2, in its version from scratch, obtained an accuracy of 98.86, 99.35 and 98.99\% respectively for the sets of tilted, proximity and mixed images. In its pre-trained version, it obtained an accuracy of 99.07 on the set of mixed images. Consequently, MobileNet V2 is ranked third in this study, but due to its speed and its small size of $14 \mathrm{MB}$, MobileNet V2 remains our team's favorite CNN for embedded applications. 


\section{CONCLUSION}

In this study, we evaluated the DL combined with the CNNs technologies in the detection of weeds in different crops. To achieve this, we studied and compared the measured scratch and pre-trained versions of state-of-the-art CNNs performance on sets of tilted, proximity and mixed images of the different cultivated plots. According to the obtained results, all the CNNs VGG 16, VGG 19, Inception V3, Inception V4, MobileNet V1 and MobileNet V2 had very good performances in the detection of weeds. Inception V4 CNN formed from scratch obtained the highest results with an accuracy of $99.18 \%, 99.45 \%$ and $99.41 \%$ respectively on the sets of tilted, proximity and mixed images. In its pre-trained version, it had an accuracy of $99.51 \%$ on the set of mixed images. This shows the effectiveness of using the pre-trained and fine-tuned CNNs version in weeds detection.

MobileNet V2 obtained an accuracy of $98.99 \%$ and $99.07 \%$ on the mixed image sets respectively for its pre-trained version and its version from scratch. But due to its speed and small size of 14MB, MobileNet V2 remains our team's preferred CNN for embedded applications. We conclude that DL technology combined with CNNs is well suited for weed detection in several types of crops. In addition, this combination forms a reliable and inexpensive solution for carrying out localized and ultra-localized spraying of herbicides on weeds and sparing crops and areas devoid of vegetation. Thus, it can participate in increasing agricultural yields and preserving the environment. We plan to continue our research in this area for the identification of plants and their various diseases.

\section{REFERENCES}

[1] A. Tannouche, K. Sbai, M. Rahmoune, R. Agounoun, A. Rahmani, and A. Rahmani., "Real time weed detection using a boosted cascade of simple features," International Journal of Electrical and Computer Engineering (IJECE), vol. 6, no. 6, pp. 2755-2765, 2016, doi: 10.11591/ijece.v6i6.pp2755-2765.

[2] A. Tannouche et al., "A fast and efficient shape descriptor for an advanced weed type classification approach," International Journal of Electrical and Computer Engineering (IJECE), vol. 6, no. 3, pp. 1168-1175, 2016, doi: 10.11591/ijece.v6i3.pp11681175 .

[3] P. Riegler-Nurscher, J. Prankl, T. Bauer, P. Strauss, and H. Prankl, "A machine learning approach for pixel wise classification of residue and vegetation cover under field conditions," Biosystems Engineering, vol. 169, pp. 188-198, 2018, doi: 10.1016/j.biosystemseng.2018.02.011.

[4] C. Potena, D. Nardi, and A. Pretto, "Fast and accurate crop and weed identification with summarized train sets for precision agriculture,” Int. Conference on Intelligent Autonomous Systems, pp. 105-121, 2016, doi: 10.1007/978-3-319-48036-7_9.

[5] W. Guo, K. U. Rage, and S. Ninomiya, "Illumination invariant segmentation of vegetation for time series wheat images based on decision tree model," Computers and Electronics in Agriculture, vol. 96, pp. 58-66, 2013, doi: 10.1016/j.compag.2013.04.010.

[6] J. M. Guerrero, G. Pajares, M. Montalvo, J. Romeo, and M. Guijarro, "Support vector machines for crop/weeds identification in maize fields," Expert Systems with Applications, vol. 39, no. 12, pp. 11149-11155, 2012, doi: 10.1016/j.eswa.2012.03.040

[7] L. Zheng, J. Zhang, and Q. Wang, "Mean-shift-based color segmentation of images containing green vegetation," Computers and Electronics in Agriculture, vol. 65, no. 1, pp. 93-98, 2009, doi: 10.1016/j.compag.2008.08.002.

[8] G. Ruiz-Ruiz, J. Gómez-Gil, and L. M. Navas-Gracia, "Testing different color spaces based on hue for the environmentally adaptive segmentation algorithm (EASA)," Computers and Electronics in Agriculture, vol. 68, no. 1, pp. 88-96, 2009, doi: 10.1016/j.compag.2009.04.009.

[9] D. A. Kumar and P. Prema, "A novel wrapping curvelet transformation based angular texture pattern (WCTATP) extraction method for weed identification," ICTACT Journal on Image and Video Processing, vol. 6, no. 3, 2016, doi: 10.21917/ijivp.2016.0174.

[10] X. Bai et al., "Vegetation segmentation robust to illumination variations based on clustering and morphology modelling," Biosystems Engineering, vol. 125, pp. 80-97, 2014, doi: 10.1016/j.biosystemseng.2014.06.015.

[11] A. Wang, W. Zhang, and X. Wei, "A review on weed detection using ground-based machine vision and image processing techniques," Computers and electronics in agriculture, vol. 158, pp. 226-240, 2019, doi: 10.1016/j.compag.2019.02.005.

[12] K. G. Liakos, P. Busato, D. Moshou, S. Pearson, and D. Bochtis, "Machine learning in agriculture: A review," Sensors, vol. 18, no. 8 , pp. 2674,2018 , doi: $10.3390 / \mathrm{s} 18082674$.

[13] M. A. I. Aquil and W. H. W. Ishak, "Evaluation of scratch and pre-trained convolutional neural networks for the classification of Tomato plant diseases," IAES International Journal of Artificial Intelligence (IJ-AI), vol. 10, no. 2, pp. 467-475, 2021, doi: 10.11591/ijai.v10.i2.pp467-475.

[14] S. Z. M. Zaki, M. A. Zulkifley, M. M. Stofa, N. A. M. Kamari, and N. A. Mohamed, "Classification of tomato leaf diseases using MobileNet v2," IAES Int. J. of Artificial Intelligence (IJ-AI), vol. 9, no. 2, pp. 290-296, 2020, doi: 10.11591/ijai.v9.i2.pp290-296.

[15] M. A. Aliyu, M. M. M. Mokji, and U. U. Sheikh, "Machine learning for plant disease detection: an investigative comparison between support vector machine and deep learning," IAES International Journal of Artificial Intelligence (IJ-AI), vol. 9, no. 4, pp. 670-683, 2020, doi: 10.11591/ijai.v9.i4.pp670-683.

[16] A. Krizhevsky, I. Sutskever, and G. E. Hinton, "Imagenet classification with deep convolutional neural networks," Advances in neural information processing systems, vol. 60, no. 6, pp. 84-90, 2017, doi: 10.1145/3065386.

[17] K. Simonyan and A. Zisserman, "Very deep convolutional networks for large-scale image recognition," 2014, arXiv preprint arXiv:1409.1556.

[18] C. Szegedy et al., "Going deeper with convolutions," IEEE Conference on Computer Vision and Pattern Recognition (CVPR), 2015, pp. 1-9, doi: 10.1109/CVPR.2015.7298594.

[19] A. G. Howard et al., "MobileNets: Efficient convolutional neural networks for mobile vision applications," 2017, arXiv preprint arXiv:1704.0486, 2017

[20] S. Ioffe and C. Szegedy, "Batch normalization: Accelerating deep network training by reducing internal covariate shift," ICML'15: Proc. of the 32nd Int. Conference on International Conference on Machine Learning, vol. 37, pp. 448-456, 2015. 
[21] C. Szegedy, V. Vanhoucke, S. Ioffe, J. Shlens, Z. Wojna, "Rethinking the inception architecture for computer vision," Proceedings of the IEEE conference on computer vision and pattern recognition, 2016, pp. 2818-2826.

[22] C. Szegedy, S. Ioffe, V. Vanhoucke, and A. A. Alemi, "Inception-v4, inception-resnet and the impact of residual connections on learning," Proceedings of the AAAI Conference on Artificial Intelligence, 2017.

[23] B. Yoo, Y. Choi, and H. Choi, "Fast depthwise separable convolution for embedded systems," International Conference on Neural Information Processing, 2018, pp. 656-665.

[24] L. Sifre and S. Mallat, "Rigid-motion scattering for texture classification," 2014, arXiv preprint arXiv:1403.1687.

[25] M. Sandler, A. Howard, M. Zhu, A. Zhmoginov, and L.-C. Chen, "MobileNetv2: Inverted residuals and linear bottlenecks," Proceedings of the IEEE conference on computer vision and pattern recognition, 2018, pp. 4510-4520.

\section{BIOGRAPHIES OF AUTHORS}

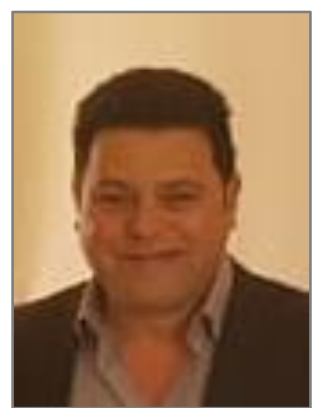

Adil Tannouche (D) SC P is a professor at the Higher School of Technology, Béni Mellal, Sultan Moulay Slimane University, Morocco. He received his PhD in electronics and embedded systems from Moulay Ismail University, Mèknes, Morocco. He is member of Laboratory of Engineering and Applied Technologies and his research area includes machine vision, artificial intelligence, and applications in the field of precision agriculture and agroindustry.Email: tannouche@gmail.com.

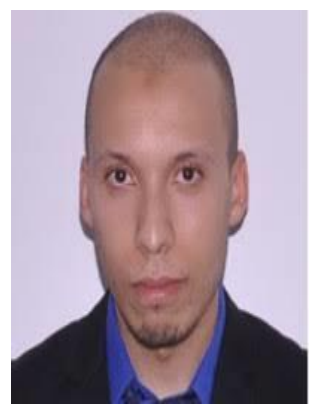

Ahmed Gaga (iD ) 8d SC P received his Engineer degree in electronic and embedded systems from university of sciences and technologies, Fez, Morocco in 2013, he was a research professor at UPF University in Fez between 2015 and 2018. Currently, he is an Assistant Professor at USMS University, Beni Mellal, Morocco. He is with Research team in Electronics, Instrumentation and Measurements, Department of Physics, He works in Electrical Engineering, especially renewable energies, and intelligent systems. His research interest is Electronics; Microcontroller based embedded systems, Photovoltaic applications, and Control theory. Email: gaga.ahmad@gmail.com.

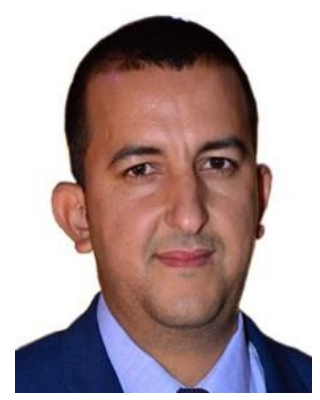

Mohammed Boutalline (iD SC SC $\mathrm{P}$ is currently an Assistant Professor in the National School of Applied Sciences of Béni Mellal at the Sultan Moulay Slimane University in Morocco. Dr Boutalline received his undergraduate degrees as well as his bachelor's degree from Sidi Mohamed Ben Abdellah University in 2009 and his master's and doctorate degrees in computer science from Sultan Moulay Slimane University (2009-2016). His research activities are currently focused on fields: computer science, big data, machine/deep learning. In addition to his scientific career, Dr Mohammed participates in the organization of several conferences namely International Conference on Optimization and Applications (ICOA), International Conference on Business Intelligence (CBI). He can be contacted at email: boutalline@gmail.com.

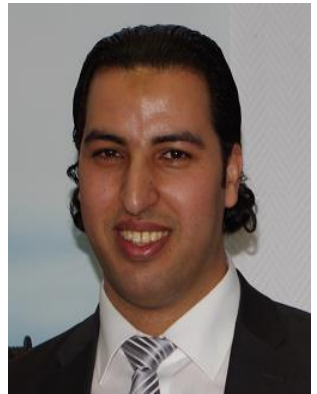

Soufiane Belhouideg (D) 8C SC P is an Associate Professor in the Department of Physics at Polydisciplinary Faculty Beni Mellal Morocco, where he has been since 2015. He received an engineering degree in Mechanical Engineering from Mohammadia School of Engineering Rabat - Morocco in 2007, and a Research Master in Behavior of Materials and Structures optimization from Polytech Annecy-Chambery School - France in 2009. He received his $\mathrm{Ph} . \mathrm{D}$. in mechanical and materials from the University of Grenoble France in 2013. He has published about fifteen research papers in international journals and more than twenty-two papers in conference proceedings. His current research focuses on additive manufacturing, smart composite structures, homogenization techniques and flow in porous media. He can be contacted at email: soufianebelhouideg@gmail.com. 